\title{
Improving the purity of one- and two-qubit gates
}

\author{
Sigmund Kohler* and Peter Hänggi** \\ Institut für Physik, Universität Augsburg, Universitätsstraße 1, 86135 Augsburg, Germany
}

Published online 4 August 2006

Key words Quantum computation, decoherence, driven systems PACS 03.67.Pp, 05.40.-a, 42.50.Hz, 03.65.Yz

We investigate the influence of AC driving fields on the coherence properties of one- and two-qubit gate operations. In both cases, we find that for suitable driving parameters, the gate purity improves significantly. A mapping of the time-dependent system-bath model to an effective static model provides analytical results. The resulting purity loss compares favorably with numerical results.

(c) 2006 WILEY-VCH Verlag GmbH \& Co. KGaA, Weinheim

\section{Introduction}

The experimental realization of one-qubit gates in solid state setups [1-3] and two-qubit gates in ion traps [4, 5] and Josephson junctions [6] has demonstrated that these systems provide remarkable coherence properties although the goal of $10^{-5}$ errors per gate operation [7] has not yet been accomplished experimentally. The unavoidable coupling to external degrees of freedom and the thereby caused decoherence still presents a main obstacle for the realization of a quantum computer. Several proposals to overcome the ensuing decoherence have been put forward, such as the use of decoherence free subspaces [8-12], coherence-preserving qubits [13], quantum Zeno subspaces [14], optimized pulse sequences [15,16], dynamical decoupling [17-21], and coherent destruction of tunneling [22,23]. Theoretical studies of decoherence of two-level systems have been extended to gate operations in the presence of an environment in [24-29].

A variety of suggestions towards coherence stabilization relies on the influence of external fields. One of the most prominent examples is the application of a sequence of $\pi$-pulses that flip the sign of the qubit-bath coupling operator resulting in a so-called dynamical decoupling (DD) of the qubit from the bath [17-21,23]. A drawback of this scheme is the fact that it eliminates only noise sources with a frequency below the repetition rate of the pulses. This clearly causes practical limitations. However, these limitations may be circumvented by using a related scheme based on continuous-wave driving, i.e. one with a harmonic timedependence, which allows higher driving frequencies.

A different proposal for coherence stabilization is to employ the physics of the so-called coherent destruction of tunneling (CDT). CDT has originally been discovered in the context of tunneling in a driven bistable potential [30-34]. There, it has been found that a particle which is initially in the, say, left well of a symmetric bistable potential, can be prevented from tunneling by the purely coherent influence of an oscillating driving field. This effect is stable against dissipation in the sense that the AC field also decelerates the dissipative transitions from the left to the right well [35-37]. Moreover, if each well of the bistable potential is contacted to a respective electron reservoir, CDT becomes visible in current measurements $[38,39]$.

A less frequently studied problem is the extension of coherence stabilization protocols to systems that consist of two or more interacting qubits. Such an interaction is essential for two-qubit operations which

* Corresponding author E-mail: sigmund.kohler@physik.uni-augsburg.de

** E-mail: peter.hanggi@physik.uni-augsburg.de 
represent an indispensable part of all quantum algorithms [40-42]. For a CNOT gate based on an isotropic Heisenberg interaction [24,43], it has been proposed to stabilize coherence by applying a control field to one of the qubits and thereby obtain an effective Ising interaction which is less sensitive to the influence of environmental degrees of freedom [44]. This scheme possesses the beneficial properties that (i) it involves only intermediately large driving frequencies that can lie well below the bath cutoff and (ii) it does not increase the gate operation time. Moreover, since the driving field couples to the same coordinate as the quantum noise, this coherence stabilization is distinctly different from the recently measured dynamical decoupling of a spin pair from surrounding spin pairs [45].

In this work, we extend our previous studies on coherence stabilization of one- [23] and two-qubit operations [44] and, moreover, detail some technical aspects. In Sect. 2, we introduce a model for two qubits coupled to a heat bath and derive a Bloch-Redfield master equation to describe quantum dissipation and decoherence. This formalism is applied in Sects. 3 and 4, respectively, to the single qubit dynamics and to a two-qubit gate operation. In each case, we derive within a rotating-wave approximation (RWA) analytical results for the decay of the gate purity. Thereby, the proper treatment of the qubit-bath coupling is of crucial importance. The computation of averages for the ensemble of all pure states is deferred to the appendix.

\section{Quantum gate with bit-flip noise}

We consider a pair of qubits described by the Hamiltonian [24,40-43]

$$
H_{\text {qubits }}=\frac{1}{2} \sum_{j=1,2}\left(\Delta_{j} \sigma_{j}^{z}+\epsilon_{j} \sigma_{j}^{x}\right)+J \vec{\sigma}_{1} \cdot \vec{\sigma}_{2},
$$

with a qubit-qubit coupling of the Heisenberg type, where $j$ labels the qubits. In order to construct a quantum gate, the tunnel splittings $\Delta_{j}$, the biases $\epsilon_{j}$, and the qubit-qubit coupling $J$ have to be controllable in the sense that they can be turned off and that their signs can be changed.

The bit-flip noise is specified by the system-bath Hamiltonian [46,47]

$$
H=H_{\text {qubits }}+H_{\text {coupl }}+H_{\text {bath }}
$$

where

$$
H_{\text {coupl }}=\frac{1}{2} \sum_{j=1,2} \sigma_{j}^{x} \sum_{\nu} \hbar c_{\nu}\left(a_{j \nu}^{\dagger}+a_{j \nu}\right)
$$

denotes the coupling of qubit $j$ to a bath of harmonic oscillators with frequencies $\omega_{\nu}$ described by the Hamiltonian $H_{\text {bath }}=\sum_{j \nu} \hbar \omega_{\nu} a_{j \nu}^{\dagger} a_{j \nu}$ and the spectral density $I(\omega)=\pi \sum_{\nu} c_{\nu}^{2} \delta\left(\omega-\omega_{\nu}\right)$. This coupling of each qubit to an individual bath represents a proper model for sufficiently distant qubits [29]. Within the present work, we consider the so-called ohmic spectral density

$$
I(\omega)=2 \pi \alpha \omega \mathrm{e}^{-\omega / \omega_{c}}
$$

with the dimensionless coupling strength $\alpha$ and the cutoff frequency $\omega_{c}$. In order to complete the model, we specify the initial condition of the Feynman-Vernon type, i.e., initially, the bath is in thermal equilibrium and uncorrelated with the system, $\rho_{\text {tot }}\left(t_{0}\right)=\rho\left(t_{0}\right) \otimes R_{\text {bath,eq }}$, where $\rho$ denotes the reduced density operator of the two qubits and $R_{\text {bath,eq }} \propto \exp \left(-H_{\mathrm{bath}} / k_{\mathrm{B}} T\right)$ is the canonical ensemble of the bath.

\subsection{Bloch-Redfield master equation}

If $\alpha k_{\mathrm{B}} T$ is smaller than the typical system energy and if the dissipation strength is sufficiently small, $\alpha \ll 1$, the dissipative system dynamics is well described within a Bloch-Redfield approach, which is also referred 
to as Born-Markov approach. There, one starts from the Liouville-von Neumann equation $\mathrm{i} \hbar \dot{\rho}_{\text {tot }}=\left[H, \rho_{\text {tot }}\right]$ for the total density operator and obtains by standard techniques the master equation [48]

$$
\begin{aligned}
\dot{\rho} & =-\frac{\mathrm{i}}{\hbar}\left[H_{\text {qubits }}, \rho\right]-\sum_{j}\left[\sigma_{j}^{x},\left[Q_{j}(t), \rho\right]\right]-\sum_{j}\left[\sigma_{j}^{x},\left\{P_{j}(t), \rho\right\}\right] \\
& \equiv-\frac{\mathrm{i}}{\hbar}\left[H_{\text {qubits }}, \rho\right]-\Lambda(t) \rho
\end{aligned}
$$

with the anti-commutator $\{A, B\}=A B+B A$ and

$$
\begin{aligned}
Q_{j}(t) & =\frac{1}{4 \pi} \int_{0}^{\infty} \mathrm{d} \tau \int_{0}^{\infty} \mathrm{d} \omega \mathcal{S}(\omega) \cos (\omega \tau) \widetilde{\sigma}_{j}^{x}(t-\tau, t), \\
P_{j}(t) & =\frac{1}{4 \pi} \int_{0}^{\infty} \mathrm{d} \tau \int_{0}^{\infty} \mathrm{d} \omega I(\omega) \sin (\omega \tau) \widetilde{\sigma}_{j}^{x}(t-\tau, t)
\end{aligned}
$$

Thus, the influence of the bath is determined by the Heisenberg operators of the system, the spectral density $I(\omega)$ of the heat baths, and the Fourier transformed

$$
\mathcal{S}(\omega)=I(\omega) \operatorname{coth}\left(\hbar \omega / 2 k_{\mathrm{B}} T\right)
$$

of the symmetrically-ordered equilibrium auto-correlation function $\frac{1}{2}\left\langle\left\{\xi_{j}(\tau), \xi_{j}(0)\right\}\right\rangle_{\text {eq }}$ of the collective bath coordinate $\xi_{j}=\sum_{\nu} c_{\nu}\left(a_{j \nu}^{\dagger}+a_{j \nu}\right)$. The notation $\widetilde{X}\left(t, t^{\prime}\right)$ is a shorthand for the Heisenberg operator $U^{\dagger}\left(t, t^{\prime}\right) X U\left(t, t^{\prime}\right)$ with $U$ being the propagator of the coherent system dynamics. Note that $\mathcal{S}(\omega)$ and $I(\omega)$ are independent of $j$ due to the assumption of two identical environments. We emphasize that the particular form (5) of the master equation is valid also for an explicitly time-dependent qubit Hamiltonian.

\subsection{Purity decay}

The heat baths, whose influence is described by the second and third term of the master equation (5), lead to decoherence, i.e., the evolution from a pure state to an incoherent mixture. This process can be measured by the decay of the purity $\operatorname{tr}\left(\rho^{2}\right)$ from the ideal value 1 . The gate purity (frequently also referred to as "purity") $\mathcal{P}(t)=\overline{\operatorname{tr}\left(\rho^{2}(t)\right)}$, which characterizes the gate independently of the specific input, results from the ensemble average over all pure initial states [49]. For weak dissipation, the purity is determined by its decay rate at initial time,

$$
\Gamma \equiv-\left.\frac{\mathrm{d}}{\mathrm{d} t} \ln \mathcal{P}(t)\right|_{t=0}=2 \overline{\operatorname{tr}(\rho \Lambda \rho)}=\frac{4}{N(N+1)} \sum_{j} \operatorname{tr}\left(\sigma_{j}^{x} Q_{j}(0)\right) .
$$

In order to obtain the last expression, we have used the cyclic property of the trace and performed the ensemble average over all pure states as described in Appendix A. Here, $N$ is the dimension of the system Hilbert space. The purity loss rate $\Gamma$ represents a figure of merit for the coherence of the quantum gate ideally, it vanishes. Interestingly enough, only the second term on the right-hand side of the master equation (5) contributes to the purity decay. This relates to the interpretation that the first term of the master equation is responsible for the coherent dynamics, while the second and third term correspond to decoherence and relaxation, respectively.

The average over all pure states, which underlies the rate (10), may differ from the average over a discrete set of initial states by a factor of the order unity, due to the particular choice of a discrete ensemble: There, one commonly chooses ensembles which are unsymmetric on the Bloch sphere $[27,28]$ or which do not include entangled states [27-29]. 


\subsection{Numerical solution}

The purity decay rate (10) by construction accounts only for the behavior at initial time $t=0$. Thus, for a more complete picture, it is desirable to have the exact numerical solution of the master equation (5) at hand. For studying the influence of an external AC field, such a solution must properly capture the case of a $T$ periodic system Hamiltonian. An efficient scheme for that purpose is a modified Bloch-Redfield formalism whose cornerstone is a decomposition into the Floquet basis of the driven system [48]: According to the Floquet theorem, the Schrödinger equation of a driven quantum system with a Hamiltonian of the form $H(t)=H(t+T)$ possesses a complete set of solutions of the form $\left|\psi_{\alpha}(t)\right\rangle=\exp \left(-\mathrm{i} \epsilon_{\alpha} t / \hbar\right)\left|\phi_{\alpha}(t)\right\rangle$. The so-called Floquet states $\left|\phi_{\alpha}(t)\right\rangle$ obey the time-periodicity of the Hamiltonian and $\epsilon_{\alpha}$ denotes the so-called quasienergy. The Floquet states are elements of an Hilbert space extended by a $T$-periodic time coordinate and are computed from the eigenvalue equation

$$
\left(H(t)-\mathrm{i} \hbar \frac{\mathrm{d}}{\mathrm{d} t}\right)\left|\phi_{\alpha}(t)\right\rangle=\epsilon_{\alpha}\left|\phi_{\alpha}(t)\right\rangle .
$$

In the Floquet basis $\left\{\left|\phi_{\alpha}(t)\right\rangle\right\}$, the master equation (5) assumes the form

$$
\dot{\rho}_{\alpha \beta}=-\frac{\mathrm{i}}{\hbar}\left(\epsilon_{\alpha}-\epsilon_{\beta}\right) \rho_{\alpha \beta}-\sum_{\alpha^{\prime} \beta^{\prime}} \Lambda_{\alpha \beta, \alpha^{\prime} \beta^{\prime}}(t) \rho_{\alpha^{\prime} \beta^{\prime}} .
$$

Besides computational advantages, using the Floquet basis has the benefit that it implies the numerically exact treatment of the coherent system dynamics. Thereby, one avoids artefacts like the violation of equilibrium properties in the undriven limit [50-54].

For weak dissipation, we can replace within a rotating-wave approximation $\Lambda(t)$ by its time average [48]. Finally, we integrate the master equation to obtain the dissipative propagator $\mathcal{W}_{\alpha \beta, \alpha^{\prime} \beta^{\prime}}$ which provides the final state $\rho_{\text {out }, \alpha \beta}=\sum_{\alpha^{\prime} \beta^{\prime}} \mathcal{W}_{\alpha \beta, \alpha^{\prime} \beta^{\prime}} \rho_{\mathrm{in}, \alpha^{\prime} \beta^{\prime}}$ and, thus, allows one to evaluate all quantities of interest. In particular, it is possible to compute for a pure initial state $|\psi\rangle=\sum_{\alpha} c_{\alpha}\left|\phi_{\alpha}\right\rangle$, i.e. for $\rho_{\text {in, } \alpha \beta}=c_{\alpha} c_{\beta}^{*}$, the final state $\rho_{\text {out }}$ which possesses the purity

$$
\operatorname{tr} \rho_{\text {out }}^{2}=\sum_{\alpha \beta} \rho_{\text {out }, \alpha \beta} \rho_{\text {out }, \beta \alpha}=\sum_{\alpha \beta \alpha^{\prime} \beta^{\prime} \alpha^{\prime \prime} \beta^{\prime \prime}} \mathcal{W}_{\alpha \beta, \alpha^{\prime} \beta^{\prime}} \mathcal{W}_{\beta \alpha, \beta^{\prime \prime} \alpha^{\prime \prime}} c_{\alpha^{\prime}} c_{\beta^{\prime}}^{*} c_{\beta^{\prime \prime}} c_{\alpha^{\prime \prime}}^{*}
$$

In order to average over all pure initial states, we employ Eq. (48) derived in the Appendix to obtain the gate purity

$$
\mathcal{P}_{\text {out }}=\frac{1}{N(N+1)} \sum_{\alpha \beta \alpha^{\prime} \beta^{\prime}}\left(\mathcal{W}_{\alpha \beta, \alpha^{\prime} \beta^{\prime}} \mathcal{W}_{\beta \alpha, \beta^{\prime} \alpha^{\prime}}+\mathcal{W}_{\alpha \beta, \alpha^{\prime} \alpha^{\prime}} \mathcal{W}_{\beta \alpha, \beta^{\prime} \beta^{\prime}}\right)
$$

We emphasize that this result is independent of the particular choice of the basis.

\section{Coherence stabilization for single qubits}

During the stage of single qubit operations, i.e., for $J=0$, both qubits together with the respective bath evolve independently of each other. Thus, it is sufficient to focus on qubit 1 with the Hilbert space dimension $N=2$. We restrict ourselves to an operation with $\epsilon_{1}=0$ and the fixed tunnel splitting $\Delta_{1}>0$, i.e. to the Hamiltonian

$$
H_{\mathrm{qubit} 1}=\frac{\Delta_{1}}{2} \sigma_{1}^{z}
$$

This situation is characterized by the fact that the bath couples to a system operator which is different from the qubit Hamiltonian. In the Bloch sphere representation sketched in Fig. 1, the qubit Hamiltonian (15) 


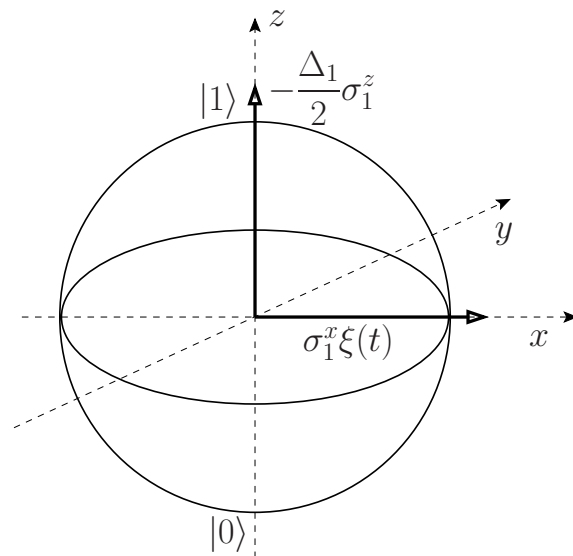

Fig. 1 Bloch sphere representation of the single-qubit Hamiltonian (15) and the bath coupling studied in Sect. 3 .

and the bath coupling correspond to orthogonal vectors. We start out by bringing the master equation (5) into a more explicit form by inserting the Heisenberg operator

$$
\tilde{\sigma}_{1}^{x}(t-\tau, t)=\sigma_{1}^{x} \cos \frac{\Delta_{1} \tau}{\hbar}+\sigma_{1}^{y} \sin \frac{\Delta_{1} \tau}{\hbar}
$$

which is readily derived from its definition together with the qubit Hamiltonian (1). Performing the integration over $\tau$ and $\omega$ and neglecting renormalization effects, which are small provided that $\alpha \ln \left(\omega_{c} / \Delta_{1}\right) \ll 1$, yields for $\Delta_{1} \ll \omega_{c}$, the Markovian master equation

$$
\dot{\rho}=-\frac{\mathrm{i}}{\hbar}\left[H_{\text {qubit1 }}, \rho\right]-\frac{\mathcal{S}\left(\Delta_{1} / \hbar\right)}{8}\left[\sigma_{1}^{x},\left[\sigma_{1}^{x}, \rho\right]\right]+\mathrm{i} \frac{\pi \alpha \Delta_{1}}{4}\left[\sigma_{1}^{x},\left\{\sigma_{1}^{y}, \rho\right\}\right] .
$$

Then the purity decay (10) is readily evaluated to read

$$
\Gamma_{0}=\frac{1}{6} \mathcal{S}\left(\Delta_{1} / \hbar\right)
$$

where the subscript " 0 " refers to the absence of any AC field.

A possible coupling to an external driving field might have any "direction" $\vec{n}$ on the Bloch sphere (cf. Fig. 1), i.e. be proportional to $\vec{n} \cdot \vec{\sigma}_{1}$. Herein, we restrict ourselves to the cases parallel to the bath coupling and parallel to the static Hamiltonian, i.e., to a field that couples to $\sigma_{1}^{z}$ and $\sigma_{1}^{x}$, respectively.

\subsection{Dynamical decoupling by harmonic driving}

The first option is to act on the qubit with a driving of the form

$$
H_{\|}=\frac{A}{2} \sigma_{1}^{z} \cos (\Omega t)
$$

"parallel" to the static Hamiltonian. This relates to a recently proposed mechanism for coherence stabilization, namely the so-called dynamical decoupling (DD) [17-21]. This scheme employs sequences of $\pi$-pulses that flip the sign of the operator $\sigma_{1}^{x}$ which couples the qubit to the bath. The basic idea dates back to the suppression of spin diffusion in nuclear magnetic resonance experiments $[55,56]$ and by now is an established technique in that area [57]. In the present case where the bath couples to the operator $\sigma_{1}^{x}$ [cf. Eq. (3)], such a transformation is e.g. induced by the Hamiltonian $\hbar \omega_{\mathrm{R}} \sigma_{1}^{z}$ for a pulse duration $\pi / \omega_{\mathrm{R}}$. Since the corresponding propagator is a function of the qubit Hamiltonian, the coherent dynamics is not altered. Besides the prospective benefits of such a control scheme, there is also a number of possible drawbacks that the application of $\pi$-pulses might cause: For a driven system, there is always the possibility of unwanted 
off-resonant transitions [58], especially in the case of ideal rectangular pulses. A more practical limitation is the fact that only noise with frequencies below the pulse repetition rate can be eliminated in such a way. These disadvantages can be overcome partially by applying a continuous wave version of the dynamical decoupling scheme, i.e. a driving of the form (19) for which the available frequency range is larger.

For the computation of the coherence properties, we use the fact that $H_{\|}(t)$ commutes with the static qubit Hamiltonian (15) and, consequently, the propagator for the driven qubit can be computed exactly reading

$$
U\left(t, t^{\prime}\right)=\exp \left(-\mathrm{i} \frac{A}{2 \hbar \Omega}\left[\sin (\Omega t)-\sin \left(\Omega t^{\prime}\right)\right] \sigma_{1}^{z}\right) \exp \left(-\frac{\mathrm{i}}{\hbar} \Delta_{1} \sigma_{1}^{z}\left(t-t^{\prime}\right)\right) .
$$

We have written the propagator in a form that is suitable for simplifying the master equation (5). Inserting this into the expression (7) results for $\Delta_{1} \ll \Omega$ in the effective coupling operator

$$
Q_{\mathrm{DD}}=\frac{1}{8}\left(J_{0}^{2}(A / \hbar \Omega) \mathcal{S}\left(\Delta_{1} / \hbar\right)+2 \sum_{n=1}^{\infty} J_{n}^{2}(A / \hbar \Omega) \mathcal{S}(n \Omega)\right) \sigma_{1}^{x} .
$$

In order to derive this expression, we have decomposed the exponentials of the trigonometric functions into a Fourier series using the identity $\exp [\mathrm{i} x \sin (\Omega t)]=\sum_{k} J_{k}(x) \exp (\mathrm{i} k \Omega t)$, where $J_{k}$ is the $k$ th order Bessel function of the first kind [59]. The effective coupling operator $Q_{\mathrm{DD}}$ is proportional to $\sigma_{1}^{x}$ and, thus, the master equation is again of the form (5). The only difference is that the dissipative terms have acquired the prefactor

$$
\eta_{\mathrm{DD}}=J_{0}^{2}(A / \hbar \Omega)+2 \sum_{n=1}^{\infty} \frac{n \hbar \Omega}{\Delta_{1}} \frac{\tanh \left(\Delta_{1} / 2 k_{\mathrm{B}} T\right)}{\tanh \left(n \hbar \Omega / 2 k_{\mathrm{B}} T\right)} \mathrm{e}^{-n \Omega / \omega_{c}} J_{n}^{2}(A / \hbar \Omega)
$$

which modifies the purity decay rate accordingly, $\Gamma_{\mathrm{DD}}=\eta_{\mathrm{DD}} \Gamma_{0}$. Equation (22) allows for the interpretation that now the decoherence rate depends on the spectral density of the bath at multiples of the driving frequency $\Omega$ which may be larger than the cutoff frequency $\omega_{c}$. The $\pi$-pulses applied in the original version [17] of dynamical decoupling, correspond for a continuous driving to a field amplitude that is adjusted such that $A / \hbar \Omega$ equals the first zero of the Bessel function $J_{0}$, i.e. it assumes a value $2.404825 \ldots$. Then only the sum in Eq. (22) contributes to the decoherence rate $\Gamma_{\mathrm{DD}}$. If now the driving frequency is larger than the cutoff of the spectral density, $\Omega>\omega_{c}$, decoherence is considerably reduced: For low temperatures, $k_{\mathrm{B}} T \ll \Delta_{1}$, the hyperbolic tangent in the factor (22) becomes unity and each contribution is weighted by a possibly large factor $n \hbar \Omega / \Delta_{1}$. In the high-temperature limit $k_{\mathrm{B}} T \gg \hbar \Omega$, we use $\tanh (x) \approx x$ and find that the dependence of the prefactor on $n \Omega$ cancels. This means that the dynamical decoupling scheme is especially useful for high temperatures. The physical reason for this is that the driving shifts the qubit dynamics towards high frequencies where the thermal occupation of the bath modes is negligible.

Fig. 2 compares the coherence stabilization $\eta_{\mathrm{DD}}$ as a function of the driving frequency for $A / \hbar \Omega=2.4$, i.e. close to a zero of the Bessel function $J_{0}$. It reveals that for driving frequencies well below the cutoff, the driving rather spoils the coherence. This improves with increasing driving frequency and, finally, for a high-frequency driving, $\eta_{\mathrm{DD}}$ becomes much smaller than unity corresponding to a significant coherence stabilization. The data demonstrate the particular usefulness of dynamical decoupling at high temperatures.

\subsection{Coherent destruction of tunneling}

Our second example under consideration is a driving field

$$
H_{\perp}(t)=f(t) \sigma_{1}^{x},
$$




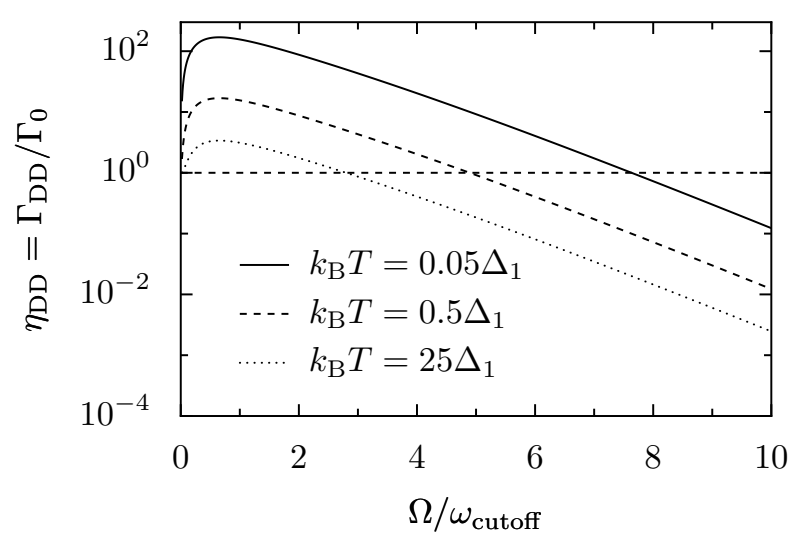

Fig. 2 Decoherence reduction by dynamical decoupling, $\eta_{\mathrm{DD}}$, as a function of the driving frequency for various temperatures. The cutoff frequency is $\omega_{c}=500 \Delta_{1} / \hbar, A / \hbar \Omega=2.4$, and the dissipation strength is $\alpha=0.01$. The horizontal line marks the value 1 below decoherence is lower than in the static case.

where the energy $f(t)$ is a $2 \pi / \Omega$-periodic function of time with zero mean. The field couples to the qubit by the same operator $\sigma_{1}^{x}$ as the bath, i.e., "perpendicular" to the static Hamiltonian (15). Thus, it commutes with the qubit-bath coupling but not with the static Hamiltonian. Such a time-dependent field causes already interesting effects for the coherent qubit dynamics that we will briefly review before discussing decoherence.

For that purpose, we derive within a rotating-wave approximation (RWA) analytical expressions for both the coherent propagator $U\left(t, t^{\prime}\right)$ and the purity decay (10). We start out by transforming the total Hamiltonian into a rotating frame with respect to the driving via the unitary transformation

$$
U_{\mathrm{AC}}(t)=\mathrm{e}^{-\mathrm{i} \phi(t) \sigma_{1}^{x}}, \quad \phi(t)=\frac{1}{\hbar} \int_{0}^{t} \mathrm{~d} t^{\prime} f\left(t^{\prime}\right) .
$$

This yields the likewise $2 \pi / \Omega$-periodic interaction-picture Hamiltonian

$$
\begin{aligned}
\widetilde{H}_{\text {qubit } 1}(t) & =U_{\text {AC }}^{\dagger}(t) H_{\text {qubit } 1} U_{\text {AC }}(t) \\
& =\frac{\Delta_{1}}{2}\left\{\sigma_{1}^{z} \cos \left(\frac{A}{\hbar \Omega} \sin (\Omega t)\right)+\sigma_{1}^{y} \sin \left(\frac{A}{\hbar \Omega} \sin (\Omega t)\right)\right\}
\end{aligned}
$$

and the S-matrix $S\left(t, t^{\prime}\right)=U_{\mathrm{AC}}^{\dagger}(t) U\left(t, t^{\prime}\right) U_{\mathrm{AC}}\left(t^{\prime}\right)$. The corresponding Schrödinger equation cannot be integrated exactly since $\widetilde{H}_{\text {qubit1 }}(t)$ does not commute with itself at different times and, thus, time-ordering has to be taken into account. We restrict ourselves to an approximate solution and neglect corrections of the order $\Delta_{1}^{2}$. Within this approximation, the propagator is simply given by the exponential of the integral of the time-dependent interaction-picture Hamiltonian. This is equivalent to replacing (26) by its time-average

$$
\bar{H}_{\text {qubit1 }} \equiv\left\langle\widetilde{H}_{\text {qubit } 1}(t)\right\rangle_{2 \pi / \Omega}=\frac{\Delta_{\text {eff }}}{2} \sigma_{1}^{z},
$$

where $\langle\ldots\rangle_{2 \pi / \Omega}$ denotes the time-average over the driving period. This RWA approximation to the driven qubit Hamiltonian is of the same form as the original static Hamiltonian (1), but with the tunneling matrix element being renormalized according to

$$
\Delta_{1} \rightarrow \Delta_{\text {eff }}=\langle\cos [2 \phi(t)]\rangle_{2 \pi / \Omega} \Delta_{1}
$$

Consequently, we find $S\left(t, t^{\prime}\right)=\exp \left\{-\mathrm{i} \bar{H}_{\text {qubit } 1}\left(t-t^{\prime}\right) / \hbar\right\}$, such that within RWA, the entire propagator for the qubit in the Schrödinger picture reads

$$
U\left(t, t^{\prime}\right)=\mathrm{e}^{-\mathrm{i} \phi(t) \sigma_{1}^{x}} \mathrm{e}^{-\mathrm{i} \bar{H}_{\text {qubit } 1}\left(t-t^{\prime}\right) / \hbar} \mathrm{e}^{\mathrm{i} \phi\left(t^{\prime}\right) \sigma_{1}^{x}} .
$$


Of particular interest are now driving parameters for which the effective tunnel splitting (28) and, thus, $\bar{H}_{\text {qubit1 }}$ vanish. Then, the one-period propagator $U(t+T, t)$ becomes the identity [recall that $U_{\mathrm{AC}}$ is $2 \pi / \Omega$ periodic and, thus, $\left.U_{\mathrm{AC}}(2 \pi / \Omega)=U_{\mathrm{AC}}(0)=1\right]$. This implies that the long-time dynamics is suppressed. The dynamics within the driving period requires a closer look at the $2 \pi / \Omega$-periodic contribution $U_{\mathrm{AC}}(t)$ : For an initial preparation in an eigenstate of $\sigma_{1}^{x}$, it provides only a global phase, such that the dynamics as a whole is suppressed also within the driving period. This effect of suppressing the time-evolution by the purely coherent influence of an external field has been investigated first in the context of driven tunneling [30,31] and is named "coherent destruction of tunneling" (CDT). Therefore, we will refer to a driving of the form (23) as "CDT driving" despite the fact that we also consider working points at which the coherent dynamics is not completely suppressed. Note that for a preparation other than an eigenstate of $\sigma_{1}^{x}$, the periodic propagator $U_{\mathrm{AC}}(t)$ will still cause a non-trivial dynamics within the driving period.

Let us now turn to the influence of the CDT driving (23) on quantum dissipation and decoherence. For that purpose, we have to evaluate the operator $Q_{j}$ contained in the master equation (5). Inserting the RWA propagator (29) into (7), we obtain after some algebra the result

$$
Q_{\mathrm{CDT}}=\frac{1}{8} \mathcal{S}\left(\Delta_{\mathrm{eff}} / \hbar\right) \sigma_{1}^{x}
$$

Thus, the master equation again is of the same form as in the undriven case, Eq. (5), but the generator of the dissipative dynamics is modified by the factor

$$
\eta_{\mathrm{CDT}}=\frac{\mathcal{S}\left(\Delta_{\text {eff }} / \hbar\right)}{\mathcal{S}\left(\Delta_{1} / \hbar\right)}=\frac{\operatorname{coth}\left(\Delta_{\text {eff }} / 2 k_{\mathrm{B}} T\right)}{\operatorname{coth}\left(\Delta_{1} / 2 k_{\mathrm{B}} T\right)}\langle\cos [2 \phi(t)]\rangle_{2 \pi / \Omega} .
$$

Consequently, the purity decay becomes $\Gamma_{\mathrm{CDT}}=\eta_{\mathrm{CDT}} \Gamma_{0}$. Since the spectral density $\mathcal{S}(\omega)$ increases monotonically with the frequency $\omega$ and, moreover, the Bessel function $J_{0}(x) \leq 1$, the CDT driving - in clear contrast to the dynamical decoupling - never enhances dissipation and decoherence.

In the high-temperature limit $k_{\mathrm{B}} T \gg \Delta_{\text {eff }}$, we employ the $\operatorname{approximation} \operatorname{coth}(x) \approx 1 / x$ which implies that for an ohmic bath, $\mathcal{S}(\Delta / \hbar) \approx 4 \pi \alpha k_{\mathrm{B}} T / \hbar$ is independent of the tunnel splitting. Consequently, $\eta_{\mathrm{CDT}} \approx 1$, i.e., the purity decay is essentially unchanged.

In the opposite limit of low temperatures, $k_{\mathrm{B}} T \ll \Delta_{\text {eff }}$, the argument of the hyperbolic cotangent is large such that $\operatorname{coth}(x) \approx 1$. Then $\eta_{\mathrm{CDT}}=\Delta_{\text {eff }} / \Delta_{1}=\langle\cos [2 \phi(t)]\rangle_{2 \pi \Omega} \leq 1$. This reduction of decoherence is brought about by the fact that the driving (23) decelerates the long time dynamics of the qubit. Thereby, the frequencies which are relevant for the decoherence are shifted to a range where the spectral density of the bath is lower. Consequently, the influence of the bath is diminished.

An important special case is that of a harmonically time-dependent driving field, $f(t)=\frac{1}{2} A \cos (\Omega t)$. Then, the time-average in the effective matrix element (28) can be expressed by a zeroth-order Bessel function of the first kind such that $\Delta_{\text {eff }}=J_{0}(A / \hbar \Omega) \Delta_{1}$. Consequently, we find that at sufficiently low temperatures decoherence is reduced by a factor $\eta_{\mathrm{CDT}}=J_{0}(A / \hbar \Omega)$. These results imply that for an ohmic bath, the coherent dynamics is slowed down by the same factor as the decoherence, cf. Eqs. (28) and (31) in the low-temperature limit. Thus, if for a specific application, the figure of merit is the number of coherent oscillations, the present coherence stabilization scheme may therefore not prove very useful.

\section{Coherence stabilization for a CNOT gate}

Logic operations in both quantum and classical computers require that the time evolution of a qubit (or a bit, respectively) depends on the state of another qubit. The physics behind such processes is a non-linear interaction between two qubits which in many implementations is of the Heisenberg type $[24,43]$

$$
H_{\text {Heisenberg }}=J \vec{\sigma}_{1} \cdot \vec{\sigma}_{2}
$$

as assumed in our model Hamiltonian (1). In the context of decoherence, the question arises whether it is possible to stabilize coherence by a proper AC field while maintaining the desired non-linear time evolution. 


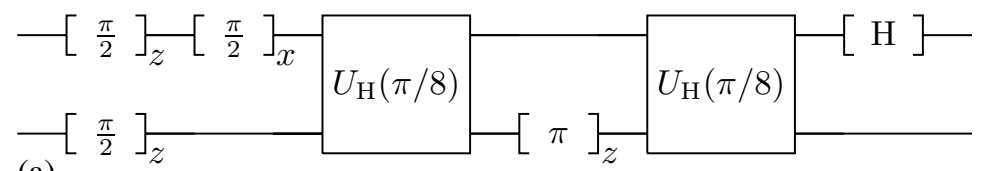

(a)

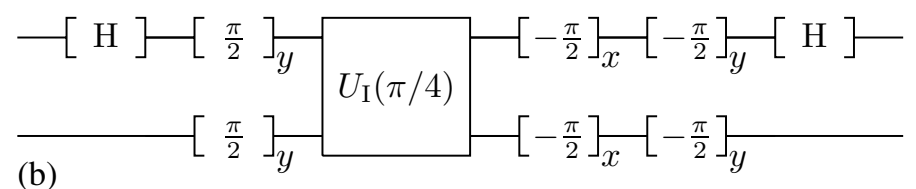

Fig. 3 Realizations of a CNOT operation for different types of qubit-qubit interaction [40-42]. (a) Heisenberg interaction providing the transformation (33) and (b) Ising coupling along the $x$-direction corresponding to (42). The symbol $[\mathrm{H}]$ denotes the Hadamard operation and $[\phi]_{n}$ the rotation of the respective qubit around the axis $n$ by an angle $\phi$.

The qubit Hamiltonian (1) together with the bath coupling (2), allows one to implement the CNOT operation sketched in Fig. 3a [24,27,40-42,60]. Apart from single qubit operations, it consists of the propagator

$$
U_{\mathrm{H}}(\varphi)=\exp \left(-\mathrm{i} \varphi \vec{\sigma}_{1} \cdot \vec{\sigma}_{2}\right)
$$

for the Heisenberg qubit-qubit interaction (32) which in total acts for a time $t_{J}=\pi \hbar / 4 J$ such that $\varphi=\pi / 4$. For single qubit operations, we have discussed in Sect. 3 that pulse sequences [17-21] and harmonic driving fields [23] can suppress decoherence. Therefore, we focus here on decoherence during the stage of the qubit-qubit interaction and, thus, take as a working hypothesis that the coherence of one-qubit operations can be stabilized ideally. Then the remaining decoherence takes place during the qubit-qubit interaction time $t_{J}$.

\subsection{Heisenberg vs. Ising coupling}

Before discussing the influence of a driving field, we provide for later reference the results for the purity decay in the static situation. Therefore, we need to evaluate $\operatorname{tr}\left(\sigma_{j}^{x} Q_{j}\right)$ where

$$
Q_{j}=\frac{1}{4 \pi} \int_{0}^{\infty} \mathrm{d} t \int_{0}^{\infty} \mathrm{d} \omega \mathcal{S}(\omega) \cos (\omega \tau) \mathrm{e}^{\mathrm{i} H_{\text {Heisenberg }} \tau / \hbar} \sigma_{j}^{x} \mathrm{e}^{-\mathrm{i} H_{\text {Heisenberg }} \tau / \hbar}
$$

This calculation is most conveniently performed in the basis of the total (pseudo) spin $\vec{L}=\frac{1}{2}\left(\vec{\sigma}_{1}+\vec{\sigma}_{2}\right)$ because $H_{\text {Heisenberg }}=J\left(2 \vec{L}^{2}-3\right)$, where $\vec{L}^{2}$ possesses the eigenvalues $\ell(\ell+1), \ell=0,1$. For $\ell=0$, the corresponding eigenstate is the singlet state $(|01\rangle-|10\rangle) / \sqrt{2}$ with energy $\epsilon_{0}=-3 J$, while for $\ell=1$, one finds the triplet $|00\rangle,(|01\rangle+|10\rangle) / \sqrt{2},|11\rangle$ with $\epsilon_{1}=J$. After evaluating the matrix elements of $\sigma_{j}^{x}$ and $Q_{j}$, we finally arrive at the purity decay rate

$$
\Gamma_{\text {Heisenberg }}=\frac{2}{5}\{\mathcal{S}(0)+\mathcal{S}(4 J / \hbar)\}
$$

The corresponding purity loss is sketched in Fig. 4. In the derivation, we have ignored Lamb shifts and defined

$$
\mathcal{S}(0) \equiv \lim _{\omega \rightarrow 0} \mathcal{S}(\omega)=\frac{4 \pi}{\hbar} \alpha k_{\mathrm{B}} T .
$$




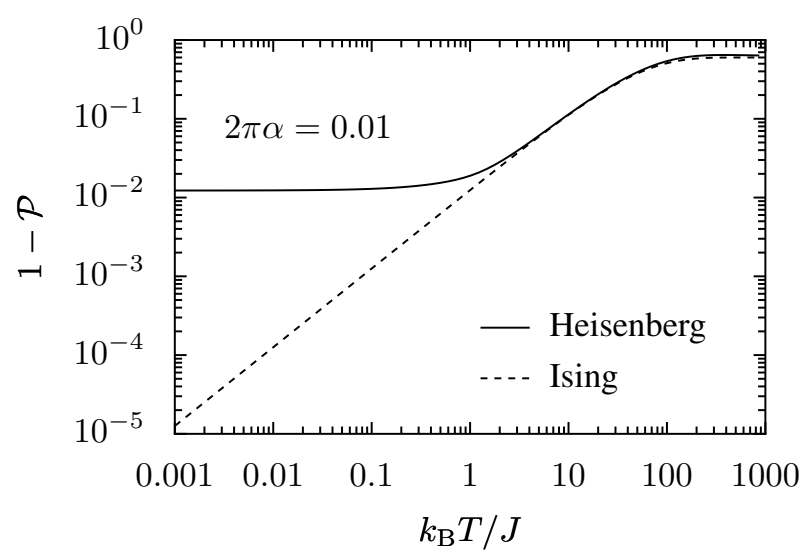

Fig. 4 Purity loss $(1-\mathcal{P})$ of two qubits interacting for a time $t_{J}=\pi \hbar / 4 J$ comparing Heisenberg (solid) and Ising (dashed) coupling. The dimensionless dissipation strength is $\alpha=0.01 / 2 \pi$.

In particular, we find that for low temperatures, $k_{\mathrm{B}} T \lesssim J$, decoherence is dominated by the term $\mathcal{S}(4 J / \hbar)$ such that $\Gamma \approx 16 \pi \alpha J / 5 \hbar$. This part reflects the influence of the so-called quantum noise which is temperature-independent and, thus, cannot be reduced by cooling the environment.

It is intriguing to compare the result (35) with the purity decay of a similar system, namely a pair of qubits interacting with the Ising interaction

$$
H_{\text {Ising }}=J \sigma_{1}^{x} \sigma_{2}^{x} \text {. }
$$

Such an interaction can also be employed for the construction of quantum gates like the CNOT gate in Fig. 3b. (Note, however, that for several physical realizations of a quantum computer [24,43], the natural interaction is of the Heisenberg type). The main difference to the Heisenberg interaction (32), is the fact that $H_{\text {Ising }}$ commutes with the bath coupling (3), i.e., the bath couples to a good quantum number. Carrying out the same calculation as above yields the purity decay

$$
\Gamma_{\text {Ising }}=\frac{4}{5} \mathcal{S}(0)=\frac{16}{5 \hbar} \pi \alpha k_{\mathrm{B}} T
$$

which exhibits a significantly different low-temperature behavior: Instead of saturating, it remains proportional to the temperature, cf. Fig. 4.

\subsection{Coherence stabilization by an AC field}

In Sects. 3.2 and 4.1 we presented two results that lead to the central idea for coherence stabilization of a qubit pair under the influence of a Heisenberg coupling: First, an AC field can suppress the dynamics "transverse" to the driving, i.e., it can effectively eliminate the parts of the Hamiltonian that depend on spin matrices other than $\sigma_{1}^{x}$. Second, qubits with Ising interaction are less sensitive to decoherence than qubits with Heisenberg interaction. Thus, the question arises whether one can act with an AC field on the system (1) in such a way that precisely the part of the Hamiltonian (32) that causes the quantum noise becomes suppressed. This is indeed the case and can be performed by driving qubit 1 with the AC field (23). Note that qubit 2 remains undriven.

For the computation of the coherence properties, we proceed as in Sect. 3.2: We first derive an effective static qubit Hamiltonian by transforming the time-dependent Hamiltonian $H_{\text {Heisenberg }}+H_{\perp}(t)$ via (24) into rotating frame and subsequently replace it by its time average. After some algebra along the lines of Sect. 3.2, we obtain the Hamiltonian

$$
\bar{H}_{\text {qubits }}=J \sigma_{1}^{x} \sigma_{2}^{x}+J_{\perp}\left(\sigma_{1}^{y} \sigma_{2}^{y}+\sigma_{1}^{z} \sigma_{2}^{z}\right),
$$

where the constant

$$
J_{\perp}=J\langle\cos [2 \phi(t)]\rangle_{2 \pi / \Omega}
$$


denotes an effective interaction "transverse" to the driving and $\langle\ldots\rangle_{2 \pi / \Omega}$ the time average over the driving period. Consequently, we find the S-matrix $S\left(t, t^{\prime}\right)=\exp \left\{-\mathrm{i} \bar{H}_{\text {qubits }}\left(t-t^{\prime}\right) / \hbar\right\}$, such that the propagator of the driven system again assumes the form (29) with $\bar{H}_{\text {qubit1 }}$ replaced by $\bar{H}_{\text {qubits }}$. Having this propagator at hand, we are in the position to derive explicit expressions for the operators $\sigma_{j}^{x}(t-\tau, t)$ and $Q_{j}$. Again, the calculation is conveniently done in the basis of the total spin $\vec{L}$ and $L_{x}$ which, owing to the relation $\sigma_{1}^{x} \sigma_{2}^{x}=\frac{1}{2}\left(\sigma_{1}^{x}+\sigma_{2}^{x}\right)^{2}-1$, is an eigenbasis of the Hamiltonian (39). We evaluate the purity decay rate (10) in this basis and finally obtain

$$
\Gamma_{\text {Heisenberg,driven }}=\frac{2}{5}\left\{\mathcal{S}(0)+\mathcal{S}\left(4 J_{\perp} / \hbar\right)\right\},
$$

i.e., the result (35) but with $J$ replaced by $J_{\perp}$. For $f(t) \equiv 0$, we find $J_{\perp}=J$ such that the static result is reproduced; otherwise, the inequality $\left|J_{\perp}\right|<J$ holds and, thus, the bath correlation function $\mathcal{S}$ in Eq. (41) has to be evaluated at a lower frequency. For an ohmic or a super-ohmic bath, $\mathcal{S}(\omega)$ is a monotonously increasing function and, consequently, the AC field reduces purity decay (unless $J>\omega_{\text {cutoff }}$ ).

The purity decay assumes its minimum for $J_{\perp}=0$. This condition marks the working points on which we shall focus henceforth. For an ohmic spectral density $I(\omega)=2 \pi \alpha \omega$, the purity decay at the working points becomes $\Gamma=\frac{4}{5} \mathcal{S}(0)=16 \pi \alpha k_{\mathrm{B}} T / 5 \hbar$. This value has to be compared to the purity decay in the absence of driving: An analysis reveals that for $k_{\mathrm{B}} T>J$, decoherence is essentially driving independent. By contrast for low temperatures, $k_{\mathrm{B}} T<J$, the driving reduces the decoherence rate by a factor $k_{\mathrm{B}} T / J$. This low-temperature behavior results from the fact that for $J_{\perp}=0$, the effective Hamiltonian (39) is identical with the Ising Hamiltonian (37) and, thus, commutes with the qubit-bath coupling operators $\sigma_{j}^{x}$. Note that the latter are not affected by the transformation (24). This means that the driving modifies the effective qubit Hamiltonian such that the bath acts as pure phase noise whose influence is proportional to the temperature. The fact that the driving field couples to the same coordinate as the bath distinguishes the present coherence stabilization from dynamical decoupling. In that respect, the present scheme is complementary to coherence-preserving qubits [13]. For the latter, heating errors are the only source of decoherence.

For a rectangular driving for which $f(t)$ switches between the values $\pm A / 2$, the condition $J_{\perp}=0$ is equivalent to $A=\hbar \Omega$ and corresponds to two $\pi$-pulses per period. For a harmonic driving, $f(t)=$ $A \cos (\Omega t) / 2$, one obtains $J_{\perp}=J J_{0}(A / \hbar \Omega)$, where $J_{0}$ denotes the zeroth-order Bessel function of the first kind. Then, at the working points $J_{\perp}=0$, the ratio $A / \hbar \Omega$ assumes a zero of $J_{0}$, i.e., one of the values $2.405 . ., 5.520 . ., 8.654 . ., \ldots .$.

So far, we ignored that the driving also affects the coherent dynamics and, thus, the pulse sequence of the CNOT operation needs a modification: At the working points of the driven system, the propagator becomes $U_{\text {eff }}\left(t, t^{\prime}\right)=U_{\mathrm{I}}\left(J\left(t-t^{\prime}\right) / \hbar\right)$ where

$$
U_{\mathrm{I}}(\varphi)=\exp \left(-\mathrm{i} \varphi \sigma_{1}^{x} \sigma_{2}^{x}\right)
$$

is the propagator corresponding to the ideal Ising interaction (37). This allows one to implement the alternative CNOT operation depicted in Fig. $3 \mathrm{~b}[41,42,61]$. Note that the interaction time $t_{J}=\pi \hbar / 4 J$ is the same as for the original gate operation in Fig. 3a. Since $U_{\mathrm{AC}}(2 \pi / \Omega)$ is the identity [cf. Eq. (24)], we assume for convenience that $t_{J}$ is an integer multiple of the driving period $2 \pi / \Omega$, i.e., $\Omega=8 \mathrm{~kJ} / \hbar$ with integer $k$.

\subsection{Numerical solution}

In order to confirm our analytical results, we compute the dissipative propagator as described in Sect. 2.3. Thereby, we restrict ourselves to purely harmonic driving $f(t)=A \cos (\Omega t) / 2$. The resulting purity loss during the interaction time $t_{J}$ is depicted in Fig. 5. We find that for large temperatures, $k_{\mathrm{B}} T>J$, decoherence is fairly independent of the driving. This behavior changes as the temperature is lowered: Once $k_{\mathrm{B}} T<J$, the purity loss is significantly reduced whenever the ratio $A / \hbar \Omega$ is close to a zero of the Bessel function $J_{0}$. Both observations confirm the preceding analytical estimates. The behavior at the first working point $A \approx 2.4 \hbar \Omega$ is depicted in Fig. 6a. For relatively low driving frequencies, we find the purity loss being 

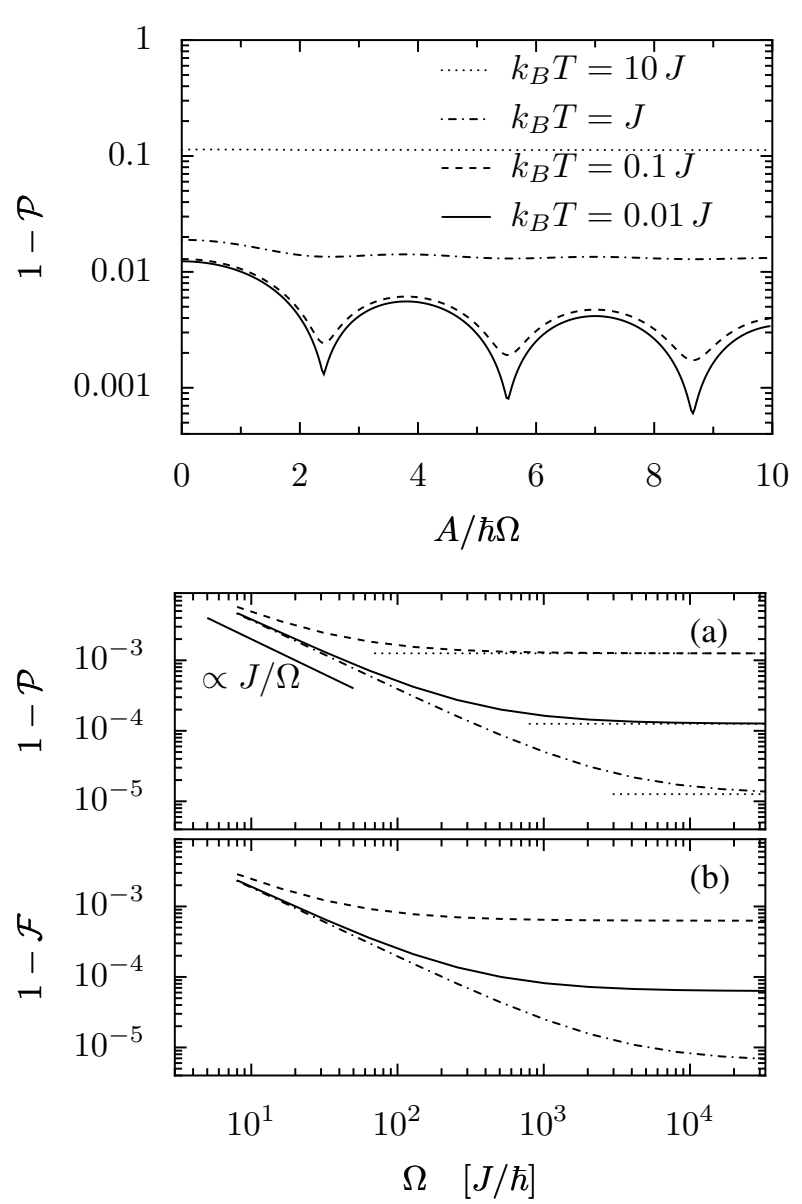

Fig. 5 Purity loss during the interaction time $t_{J}$ as a function of the driving amplitude. The driving frequency is $\Omega=32 \mathrm{~J} / \hbar$ and the dissipation strength $2 \pi \alpha=0.01$. For $A=0$, the undriven situation is reproduced.
Fig. 6 (a) Purity loss $(1-\mathcal{P})$ for a pair of qubits with Heisenberg interaction as a function of the driving frequency for the temperatures $k_{\mathrm{B}} T=0.1 \mathrm{~J}$ (dashed), 0.01J (solid), and 0.001J (dash-dotted). The dotted lines mark the analytical estimate 1 $\mathcal{P}\left(t_{J}\right) \approx \Gamma_{\text {Ising }} t_{J}$. (b) Corresponding fidelity defect $1-\mathcal{F}$.

proportional to $J / \Omega$. This significant deviation from the analytical result for small $\Omega$ relates to the fact that the low-frequency regime is not within the scope of our rotating-wave approximation which assumes $\Omega$ to be the largest frequency scale. With increasing driving frequency, the discrepancy decreases until finally decoherence is dominated by thermal noise $\propto T$ and the numerical solution confirms the analytical results. Fig. 7 reveals that the accuracy of our analytical estimates increases with the driving frequency: While for the relatively low frequency $\Omega=16 \mathrm{~J} / \hbar$, the purity loss is still close to the one of the undriven Heisenberg gate, it converges in the limit $\Omega \rightarrow \infty$ to the value obtained for Ising interaction (cf. the dashed line in Fig. 4).

Still, there remains one caveat: The gate operation in Fig. $3 b$ relies on the fact that the static effective Hamiltonian $\bar{H}_{\text {qubits }}$ describes the dynamics of the driven system sufficiently well - any discrepancy results in a coherent error. Therefore, we still have to justify that such coherent errors are sufficiently small. As a measure, we employ the so-called fidelity [49]

$$
\mathcal{F}=\overline{\operatorname{tr}\left[\rho_{\text {ideal }} \rho\left(t_{J}\right)\right]},
$$

which is defined as the overlap between the real outcome of the operation, $\rho\left(t_{J}\right)$, and the desired final state $\rho_{\text {ideal }}=U_{\mathrm{I}}(\pi / 4) \rho_{\text {in }} U_{\mathrm{I}}^{\dagger}(\pi / 4)$ in the average over all pure initial states. The time-evolution (42) of the ideal Ising qubit-qubit interaction is characterized by $\mathcal{F}=1$. Fig. 6 b demonstrates that the fidelity defect $1-\mathcal{F}$ at the first working point is even smaller than the purity loss. Thus, we can conclude that coherent errors are not of a hindrance. 


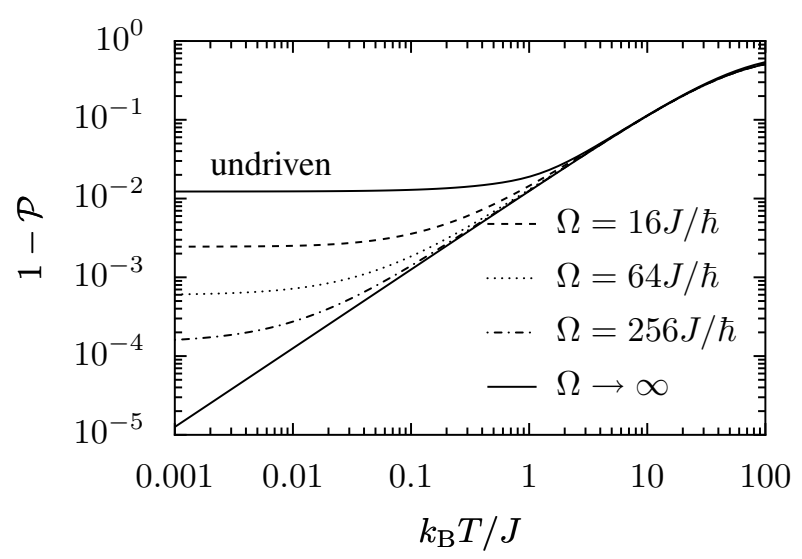

Fig. 7 Purity loss shown in Fig. 6 as a function of the temperature. The driving amplitude $A \approx 2.4 \hbar \Omega$ is adjusted such that $1-\mathcal{P}$ assumes its first minimum; cf. Fig. 5. All other parameters are as in Fig. 6.

\subsection{Implementation with quantum dots}

Fig. 7 indicates that the benefits of a "preferably infinitely" large driving frequency and amplitude. Thus, a crucial question is whether sufficiently large values are experimentally within reach. For spin qubits in quantum dots [24] a typical exchange coupling is $J=0.1 \mathrm{meV}$ which for a temperature $T=10 \mathrm{mK}$ corresponds to the solid lines in Figs. 5 and 6. These results demonstrate that driving with the feasible frequency $\Omega=2 \pi \times 100 \mathrm{~J} / \hbar \approx 10^{12} \mathrm{~Hz}$ and amplitude $A=10 \mathrm{meV}$ already reduces the purity loss by two orders of magnitude while the fidelity loss stays at a tolerable level.

\section{Conclusions}

We have investigated the influence of oscillating fields on the coherence properties of one- and two-qubit gate operations for three different cases for which it is beneficial. The first case constitutes a continuouswave version of dynamical decoupling of a single qubit from its environment. It is characterized by a driving Hamiltonian that does not commute with the bath coupling. There, we have found that a low-frequency driving is rather destructive because it generally even enforces decoherence. However, once the frequency exceeds the bath cutoff, the coherence properties recover and are finally significantly improved, especially at high temperatures. Since such a dynamical decoupling by a harmonic driving allows higher driving frequencies than the pulsed version, this form of coherence stabilization bears interesting perspectives for applications.

A second possibility for manipulating the decoherence of a single qubit is provided by the physics of coherent destruction of tunneling. For such a driving, we have found that the coherence stabilization results from the fact that the driving shifts the coherent long-time dynamics of the qubit towards lower frequencies. There, the spectral density of an ohmic bath is lower and, consequently, the effective dissipation is weaker. This implies that decoherence is generally reduced - most significantly at low temperatures. For high temperatures, however, the lower spectral density is counterbalanced by an increasing thermal noise, such that in this regime decoherence is essentially not influenced the driving.

For two qubits interacting via a Heisenberg exchange coupling, a suited AC field turns the interaction into an effective Ising interaction, which is much less sensitive to decoherence. For qubits with such a Heisenberg interaction, like e.g. spin qubits, this suggests the following coherence stabilization protocol: Use for the CNOT operation a pulse sequence that is suitable for Ising interaction with the latter being realized by a Heisenberg interaction with a proper additional AC field. This coherence stabilization scheme differs from previous proposals in two respects: First, it is different from dynamical decoupling because the driving commutes with the bath coupling. By contrast, the central idea of our scheme is to suppress rather the coherent system dynamics "transverse" to this sensitive system coordinate. Consequently, the bit-flip noise acts as pure phase noise, which is proportional to the temperature. Cooling, thus, enables a further 
coherence gain. The second difference is that the proposed scheme eliminates also the noise stemming from the spectral range above the driving frequency and, thus, is particularly valuable for ohmic noise spectra with large cutoff frequencies. Moreover, the driven system still allows one to perform the desired CNOT operation with high fidelity and within the same operation time as in the absence of the control field. Hence, the gained coherence time fully contributes to the number of feasible gate operations.

Acknowledgements We acknowledge helpful discussions with K. M. Fonseca-Romero, C. M. Wubs, R. Doll, F. K. Wilhelm, M. J. Storcz, and U. Hartmann. This work has been supported by the Freistaat Bayern via the network "Quanteninformation längs der A8" and by the Deutsche Forschungsgemeinschaft through SFB 631.

\section{A Average over all pure states}

In this appendix, we derive formulas for the evaluation of expressions of the type $\operatorname{tr}(\rho A)$ and $\operatorname{tr}(\rho A \rho B)$ in an ensemble average over all pure states $\rho=|\psi\rangle\langle\psi|$. The state $|\psi\rangle$ is an element of an $N$-dimensional Hilbert space. Decomposed into an arbitrary orthonormal basis set $\{|n\rangle\}_{n=1 \ldots N}$, it reads

$$
|\psi\rangle=\sum_{n=1}^{N} c_{n}|n\rangle
$$

where the only restriction imposed on the coefficients $c_{n}$ is the normalization $\langle\psi \mid \psi\rangle=\sum_{n}\left|c_{n}\right|^{2}=1$. Hence the ensemble of all pure states is described by the distribution

$$
P\left(c_{1}, \ldots, c_{N}\right)=\frac{2 \pi^{N}}{(N-1) !} \delta\left(1-\sum_{n=1}^{N}\left|c_{n}\right|^{2}\right)
$$

The prefactor on the right-hand side of Eq. (45) has been determined from the normalization

$$
\int \mathrm{d}^{2} c_{1} \ldots \mathrm{d}^{2} c_{N} P\left(c_{1}, \ldots, c_{N}\right)=1
$$

of the distribution and $\int \mathrm{d}^{2} c$ denotes integration over the real and the imaginary part of $c$. We emphasize that $P\left(c_{1}, \ldots, c_{N}\right)$ is invariant under unitary transformations of the state $|\psi\rangle$. The computation of the ensemble averages of the coefficients with the distribution (45) is straightforward and yields

$$
\begin{aligned}
\overline{c_{m} c_{n}^{*}} & =\frac{1}{N} \delta_{m n}, \\
\overline{c_{m} c_{n}^{*} c_{m^{\prime}} c_{n^{\prime}}^{*}} & =\frac{1}{N(N+1)}\left(\delta_{m n} \delta_{m^{\prime} n^{\prime}}+\delta_{m n^{\prime}} \delta_{n m^{\prime}}\right) .
\end{aligned}
$$

Using these expressions, we find for the ensemble averages of the expressions $\operatorname{tr}(\rho A)$ and $\operatorname{tr}(\rho A \rho B)$ the results

$$
\begin{aligned}
\overline{\operatorname{tr}(\rho A)} & =\overline{\langle\psi|A| \psi\rangle}=\frac{\operatorname{tr}(A)}{N}, \\
\overline{\operatorname{tr}(\rho A \rho B)} & =\overline{\langle\psi|A| \psi\rangle\langle\psi|B| \psi\rangle}=\frac{\operatorname{tr}(A) \operatorname{tr}(B)+\operatorname{tr}(A B)}{N(N+1)},
\end{aligned}
$$

which have been used to obtain the purity decay rate (10). 


\section{References}

[1] Y. Nakamura, Y.A. Pashkin, and J. S. Tsai, Nature 398, 786 (1999).

[2] D. Vion, A. Aassime, A. Cottet, P. Joyez, H. Pothier, C. Urbina, D. Esteve, and M. H. Devoret, Science 296, 886 (2002).

[3] I. Chiorescu, Y. Nakamura, C. J.P. Harmans, and J.E. Mooij, Science 299, 1869 (2003).

[4] D. Leibfried, B. DeMarco, V. Meyer, D. Lucas, M. Barrett, J. Britton, W. M. Itano, B. Jelenković, C. Langer, T. Rosenband, and D. J. Wineland, Nature 422, 412 (2003).

[5] F. Schmidt-Kaler, H. Häffner, M. Riebe, S. Gulde, G.P.T. Lancaster, T. Deuschle, C. Becher, C.F. Roos, J. Eschner, and R. Blatt, Nature 422, 408 (2003).

[6] Y.A. Pashkin, T. Yamamoto, O. Astafiev, Y. Nakamura, D. V. Averin, and J. S. Tsai, Nature 421, 823 (2003).

[7] A. M. Steane, Fortschr. Phys. 46, 443 (1998).

[8] G. M. Palma, K.-A. Suominen, and A. K. Ekert, Proc. R. Soc. London, Ser. A 452, 567 (1996).

[9] L.-M. Duan and G.-C. Guo, Phys. Rev. Lett. 79, 1953 (1997).

[10] P. Zanardi and M. Rasetti, Phys. Rev. Lett. 79, 3306 (1997).

[11] D. A. Lidar, I. L. Chuang, and K. B. Whaley, Phys. Rev. Lett. 81, 2594 (1998).

[12] A. Beige, D. Braun, B. Tregenna, and P. L. Knight, Phys. Rev. Lett. 85, 1762 (2000).

[13] D. Bacon, K. R. Brown, and K. B. Whaley, Phys. Rev. Lett. 87, 247902 (2001).

[14] P. Facchi and S. Pascazio, Phys. Rev. Lett. 89, 080401 (2002).

[15] H. Jirari and W. Pötz, Phys. Rev. A 72, 013409 (2005).

[16] A. K. Spoerl, T. Schulte-Herbrueggen, S. J. Glaser, V. Bergholm, M. J. Storcz, J. Ferber, and F. K. Wilhelm, quant-ph/0504202.

[17] L. Viola and S. Lloyd, Phys. Rev. A 58, 2733 (1998).

[18] L. Viola, E. Knill, and S. Lloyd, Phys. Rev. Lett. 82, 2417 (1999).

[19] D. Vitali and P. Tombesi, Phys. Rev. A 65, 012305 (2002).

[20] H. Gutmann, F. K. Wilhelm, W. M. Kaminsky, and S. Lloyd, Phys. Rev. A 71, 020302(R) (2005).

[21] G. Falci, A. D’Arrigo, A. Mastellone, and E. Paladino, Phys. Rev. A 70, 040101(R) (2004).

[22] M. Thorwart, L. Hartmann, I. Goychuk, and P. Hänggi, J. Mod. Opt. 47, 2905 (2000).

[23] K. M. Fonseca-Romero, S. Kohler, and P. Hänggi, Chem. Phys. 296, 307 (2004).

[24] D. Loss and D. P. DiVincenzo, Phys. Rev. A 57, 120 (1998).

[25] M. Grifoni, E. Paladino, and U. Weiss, Eur. Phys. J. B 10, 719 (1999).

[26] M. Governale, M. Grifoni, and G. Schön, Chem. Phys. 268, 273 (2001).

[27] M. Thorwart and P. Hänggi, Phys. Rev. A 65, 012309 (2002).

[28] M. J. Storcz and F. K. Wilhelm, Phys. Rev. A 67, 042319 (2003).

[29] M. J. Storcz, U. Hartmann, S. Kohler, and F. K. Wilhelm, Phys. Rev. B 72, 235321 (2005).

[30] F. Grossmann, T. Dittrich, P. Jung, and P. Hänggi, Phys. Rev. Lett. 67, 516 (1991).

[31] F. Großmann, P. Jung, T. Dittrich, and P. Hänggi, Z. Phys. B 84, 315 (1991).

[32] F. Großmann and P. Hänggi, Europhys. Lett. 18, 571 (1992).

[33] Y. Kayanuma, Phys. Rev. A 50, 843 (1994).

[34] M. Grifoni and P. Hänggi, Phys. Rep. 304, 229 (1998).

[35] T. Dittrich, B. Oelschlägel, and P. Hänggi, Europhys. Lett. 22, 5 (1993).

[36] M. Grifoni, M. Sassetti, P. Hänggi, and U. Weiss, Phys. Rev. E 52, 3596 (1995).

[37] L. Hartmann, I. Goychuk, M. Grifoni, and P. Hänggi, Phys. Rev. E 61, R4687 (2000).

[38] J. Lehmann, S. Camalet, S. Kohler, and P. Hänggi, Chem. Phys. Lett. 368, 282 (2003).

[39] S. Kohler, S. Camalet, M. Strass, J. Lehmann, G.-L. Ingold, and P. Hänggi, Chem. Phys. 296, 243 (2004).

[40] M.A. Nielsen and I.L. Chuang, Quantum Computing and Quantum Information (Cambridge University Press, Cambridge, 2000).

[41] Y. Makhlin, G. Schön, and A. Shnirman, Rev. Mod. Phys. 73, 357 (2001).

[42] A. Galindo and M. A. Martín-Delgado, Rev. Mod. Phys. 74, 347 (2002).

[43] B. Kane, Nature (London) 393, 133 (1998).

[44] K. M. Fonseca-Romero, S. Kohler, and P. Hänggi, Phys. Rev. Lett. 95, 140502 (2005).

[45] C. Ramanathan, S. Sinha, J. Baugh, T.F. Havel, and D. G. Cory, Phys. Rev. A 71, 020303(R) (2005).

[46] P. Hänggi, P. Talkner, and M. Borkovec, Rev. Mod. Phys. 62, 251 (1990). 
[47] A. J. Leggett, S. Chakravarty, A.T. Dorsey, M.P.A. Fisher, A. Garg, and W. Zwerger, Rev. Mod. Phys. 59, 1 (1987).

[48] S. Kohler, T. Dittrich, and P. Hänggi, Phys. Rev. E 55, 300 (1997).

[49] J.F. Poyatos, J.I. Cirac, and P. Zoller, Phys. Rev. Lett. 78, 390 (1997).

[50] E. G. Petrov, I. A. Goychuk, and V. May, Phys. Rev. E 54, 4726 (1996).

[51] E. G. Petrov, I. A. Goychuk, and V. May, Chem. Phys. Lett. 253, 428 (1996).

[52] T. Novotný, Europhys. Lett. 59, 648 (2002).

[53] V. May and O. Kühn, Charge and Energy Transfer Dynamics in Molecular Systems, 2nd ed. (Wiley-VCH, Weinheim, 2003).

[54] S. Kohler, J. Lehmann, and P. Hänggi, Phys. Rep. 406, 379 (2005).

[55] H.Y. Carr and E. M. Purcell, Phys. Rev. 94, 630 (1954).

[56] U. Haeberlen and J. S. Waugh, Phys. Rev. 175, 453 (1968).

[57] C. P. Slichter, Principles of Magnetic Resonance, Springer Series in Solid State Sciences, Vol. 1, 3rd ed. (Springer, Berlin, 1990).

[58] L. Tian and S. Lloyd, Phys. Rev. A 62, 050301 (2000).

[59] I. M. Gradshteyn and I. S. Ryzhik, Table of Integrals, Series, and Products, 5th ed. (Academic Press, San Diego, 1994).

[60] N. Schuch and J. Siewert, Phys. Rev. A 67, 032301 (2003).

[61] N. Gershenfeld and I. L. Chuang, Science 275, 350 (1997). 